\title{
Energy and angular momentum radiated for non head-on binary black hole collisions
}

\author{
Osvaldo M. Moreschi ${ }^{1 *}$ Alejandro Perez ${ }^{2}$ and Luis Lehner ${ }^{3 \dagger}$ \\ ${ }^{1}$ FaMAF, Universidad Nacional de Córdoba, \\ Ciudad Universitaria, (5000) Córdoba, Argentina. \\ ${ }^{2}$ Center for Gravitational Physics and Geometry, \\ Penn State University, University Park, PA 16802, U.S.A. \\ ${ }^{3}$ Department of Physics \& Astronomy, \\ The University of British Columbia, Vancouver BC, V6T 1Z1, Canada.
}

September 13, 2002

\begin{abstract}
We investigate the possible total radiated energy produced by a binary black hole system containing nonvanishing total angular momentum. For the scenarios considered we find that the total radiated energy does not exceed $1 \%$. Additionally we explore the gravitational radiation field and the variation of angular momentum in the process.

After the formation of the final black hole, the model uses the Robinson-Trautman (RT) spacetimes as background. The evolution of perturbed RT geometries is carried out numerically.
\end{abstract}

PACS: 04.25.Dm, 04.30.-w, 04.30.Db, 02.60.Cb

\section{Introduction}

The advent of powerful detectors capable of directly measuring gravitational radiation for the first time has motivated numerous investigations of systems likely to produce gravitational waves of enough intensity which are expected to be observed with these detectors. Among those systems, a prime candidate is that composed by a couple of inspiraling black holes which eventually merge into one releasing considerable amounts of energy via gravitational radiation. Clearly, because of the strong gravitational fields involved in such process, its complete description requires solving Einstein equations in their full generality which can only be done through numerical methods [1]. Several groups are combining efforts to numerically model such system [2, 3, 4, 5, 6, 7] and although significant progress has been (and is being) made in this direction (see for instance[1]), an accurate and robust implementation is still missing. In the mean time, valuable insight can be gained through approximate models

* Member of CONICET.

${ }^{\dagger}$ CITA National Fellow and PIMS Post-Doctoral Fellow. of the system. These models serve both as means to gain a better understanding of the problem and also to provide information that can serve as tests for the numerical simulations, where the lack of known solutions renders the problem of accuracy assessment more involved.

Traditional models are obtained through perturbative approximations where an expansion with respect to some appropriately chosen parameter provides a reduced and manageable system of equations. Naturally, these approaches provide accurate answers only when the perturbative parameter remains small. For instance, the traditional post-Newtonian approximation (see for instance 8, 9), can be safely used when the black holes are far enough and the relative speeds involved are much smaller than 1. However, as the holes come closer, the gravitational fields and relative speeds involved become very large and it is clear that this approximation will fail to give sensible answers. Resumation techniques 10 and/or effective one-body expansions [11 have been proposed to extend the regime of validity of this approach. However, even when the ambiguities proper of these options can be satisfactorily addressed, they will inevitably break in the late stages of the merger, which for the case of black holes lies in the maximum sensitivity window of earth-based detectors 12, 13.

Another approximation, known as the close limit approximation (CLA) (see for instance 14, 15]) assumes the black holes have already merged and the perturbation parameter can be considered to be how nonSchwarzschild (or non-Kerr) the hole is. Since no-hair theorems imply the final fate of the formed black hole should be of the Kerr-type, this approach can safely be used to describe such epoch (note however, that in certain situations, the CLA has been able to produce valuable results at rather earlier times when the holes had just merged; however, it is not clear that this will hold in generic cases).

A different approximate model can be obtained by 
considering Robinson-Trautman spacetimes which contain purely outgoing gravitational radiation and decay to leave a Schwarzschild-like horizon 17, 18, 19. These spacetimes are thus natural candidates to study systems settling to a single black hole. The idea here would be to use them as background spacetimes for a perturbation treatment For instance, a perturbation approach can be constructed where the perturbation parameter is the gravitational radiation produced by the system 20]. This indeed appears as an enticing suggestion since the system is not expected to produce total radiation outputs in excess of few percent of the initial mass.

In the past, this idea was pursued to produce estimates of the total gravitational radiation 21, 22 obtaining excellent agreement with numerical relativity results for the head-on collision, i.e. zero angular momentum case 23. These studies did not allow for the spacetime to have total angular momentum and also the physical situations considered produced small enough radiation output that the equations governing the dynamic of the perturbations were solved in the asymptotic late-time linear regime 21, 22].

In order to consider more interesting scenarios, perturbations allowing for angular momentum were introduced in 24] thus paving the way for studies of more generic situations. In the present work, we perform such studies by numerically solving the dynamical equations introduced in 24 in their full generality. Our studies are aimed towards obtaining a deeper understanding of the system and also with the hope of producing information that can be used as a check of full numerical relativistic simulations.

This work is organized as follows, in section 2 we briefly review the perturbative treatment of RobinsonTrautman (RT) spacetimes that allows for angular momentum. Section 3 describes the physical parameters and how to make the matching between the two eras that appear in our model. The numerical implementation to solve the equations that govern the evolution of the perturbations is described in Section 4 . The numerical results are presented in Section 5 where the total energy, the gravitational radiation field and the evolution of angular momentum are shown. We conclude with some final comments in Section 6 .

\section{General angular momentum perturbations of RT spacetimes}

In 24], generic perturbations of RT spacetimes, which include angular momentum, have been presented.

It is convenient to express the geometry in terms of a

\footnotetext{
${ }^{1}$ Note that this approach would therefore have a background spacetime which radiates as opposed to considering perturbations off a stationary spacetime.
}

null tetrad $\left(\ell^{a}, m^{a}, \bar{m}^{a}, n^{a}\right)$ where:

$$
g_{a b} \ell^{a} n^{b}=-g_{a b} m^{a} \bar{m}^{b}=1
$$

with all other possible scalar products being zero; then, the metric can be expressed by

$$
g_{a b}=\ell_{a} n_{b}+n_{a} \ell_{b}-m_{a} \bar{m}_{b}-\bar{m}_{a} m_{b}
$$

In terms of the coordinate system $\left(x^{0}, x^{1}, x^{2}, x^{3}\right)=$ $\left(u, r,(\zeta+\bar{\zeta}), \frac{1}{i}(\zeta-\bar{\zeta})\right)$, where $u$ is a null coordinate and $r$ is an affine parameter along the geodesic integral lines of the vector $\ell^{a}$, one can express the null tetrad in terms of its components by the relations:

$$
\begin{gathered}
\ell_{a}=(d u)_{a} \\
\ell^{a}=\left(\frac{\partial}{\partial r}\right)^{a} \\
m^{a}=\xi^{i}\left(\frac{\partial}{\partial x^{i}}\right)^{a} \\
\bar{m}^{a}=\bar{\xi}^{i}\left(\frac{\partial}{\partial x^{i}}\right)^{a} \\
n^{a}=U\left(\frac{\partial}{\partial r}\right)^{a}+X^{i}\left(\frac{\partial}{\partial x^{i}}\right)^{a}
\end{gathered}
$$

with $i=0,2,3$ and $\zeta=\frac{1}{2}\left(x^{2}+i x^{3}\right)$; and where the components $\xi^{i}, U$ and $X^{i}$ are given by the following expressions:

$$
\begin{gathered}
\xi^{0}=0 \\
\xi^{2}=\frac{\xi_{0}^{2}}{r}+\lambda \bar{\xi}_{0}^{2}\left(-\frac{\sigma_{0}}{r^{2}}+\frac{1}{r} \frac{\partial^{2} W_{0}}{\partial r^{2}}-\frac{2}{r^{2}} \frac{\partial W_{0}}{\partial r}\right) \\
\xi^{3}=\frac{\xi_{0}^{3}}{r}+\lambda \bar{\xi}_{0}^{3}\left(-\frac{\sigma_{0}}{r^{2}}+\frac{1}{r} \frac{\partial^{2} W_{0}}{\partial r^{2}}-\frac{2}{r^{2}} \frac{\partial W_{0}}{\partial r}\right) \\
\xi_{0}^{2}=\sqrt{2} P_{0} V, \quad \xi_{0}^{3}=-i \xi_{0}^{2} \\
U=r U_{00}+U_{0}+\frac{U_{1}}{r}+\frac{U_{2}}{r^{2}}+\Delta U_{3}
\end{gathered}
$$

$$
\begin{aligned}
U_{00} & =\frac{\dot{V}}{V} \\
U_{0} & =-\frac{1}{2} K_{V}, \\
U_{1} & =-\frac{1}{2}\left(\Psi_{2}^{0}+\bar{\Psi}_{2}^{0}\right), \\
U_{2} & =\frac{\lambda}{6}\left(\check{\partial}_{V_{R T}} \bar{\Psi}_{1}^{0}+\overline{\mathrm{\partial}}_{V_{R T}} \Psi_{1}^{0}\right), \\
\Delta U_{3} & =-\frac{\lambda}{r^{2}}\left(\bar{\partial}_{V_{R T}}^{2} W_{0}+\check{\partial}_{V_{R T}}^{2} \bar{W}_{0}\right) ;
\end{aligned}
$$




$$
\begin{aligned}
X^{0}= & 1, \\
X^{2}= & \lambda \xi_{0}^{2}\left(-\frac{\bar{\tau}_{0}}{r^{2}}+\frac{2 \bar{\Psi}_{1}^{0}}{3 r^{3}}+\frac{2}{r^{2}} \frac{\partial \oiint_{V_{R T}} \bar{W}_{0}}{\partial r}-\frac{4}{r^{3}}{\left.\partial_{V_{R T}} \bar{W}_{0}\right)}+\right.\text { c.c, } \\
X^{3}= & \lambda \xi_{0}^{3}\left(-\frac{\bar{\tau}_{0}}{r^{2}}+\frac{2 \bar{\Psi}_{1}^{0}}{3 r^{3}}+\frac{2}{r^{2}} \frac{\partial \oiint_{V_{R T}} \bar{W}_{0}}{\partial r}-\frac{4}{r^{3}}{\left.\partial_{V_{R T}} \bar{W}_{0}\right)}+\right.\text { c.c; }
\end{aligned}
$$

where

$$
\tau_{0}=\bar{\partial}_{V_{R T}} \sigma_{0},
$$

c.c means complex conjugate,

$$
K_{V}=\frac{2}{V} \bar{\partial}_{V} \partial_{V} V-\frac{2}{V^{2}} \partial_{V} V \bar{\partial}_{V} V+V^{2} ;
$$

and where in these equations we are explicitly denoting the first order terms by introducing the first order parameter $\lambda$ dependency, and where $\partial_{V}$ is the edth operator 25, 26, 27], in the GHP notation 28], of the sphere with metric

$$
d S^{2}=\frac{1}{P^{2}} d \zeta d \bar{\zeta}
$$

where $P=V(u, \zeta, \bar{\zeta}) P_{0}(\zeta, \bar{\zeta})$, and $P_{0}$ is the value of $P$ for the unit sphere.

The scalar $V$ is given by

$$
V=V_{R T}+\lambda V_{\lambda}
$$

where $V_{R T}$ is the RT scalar satisfying the RobinsonTrautman equation

$$
-3 M_{0} \dot{V}_{R T}=V_{R T}^{4} \partial^{2} \bar{\partial}^{2} V_{R T}-V_{R T}^{3} \partial^{2} V_{R T} \bar{\delta}^{2} V_{R T},
$$

$V_{\lambda}$ is the linear perturbation scalar and $\partial$ is the edth operator of the unit sphere. One can then express $K_{V}$ by

$$
K_{V}=K_{V_{R T}}+\lambda K_{V_{\lambda}},
$$

where

$$
\begin{aligned}
K_{V_{R T}}=\frac{2}{V_{R T}} & \bar{\partial}_{V_{R T}} \partial_{V_{R T}} V_{R T} \\
& \quad-\frac{2}{V_{R T}^{2}} \partial_{V_{R T}} V_{R T} \bar{\partial}_{V_{R T}} V_{R T}+V_{R T}^{2},
\end{aligned}
$$

and

$$
\begin{gathered}
K_{V_{\lambda}}=\frac{2}{V_{R T}} \bar{\partial}_{V_{R T}} \partial_{V_{R T}} V_{\lambda}-\frac{2}{V_{R T}^{2}} \varlimsup_{V_{R T}} V_{\lambda} \bar{\partial}_{V_{R T}} V_{R T} \\
-\frac{2}{V_{R T}^{2}} \partial_{V_{R T}} V_{R T} \bar{\partial}_{V_{R T}} V_{\lambda}+\frac{2 V_{\lambda}}{V_{R T}^{3}} \partial_{V_{R T}} V_{R T} \bar{\partial}_{V_{R T}} V_{R T} \\
+V_{\lambda} V_{R T}+\frac{V_{\lambda}}{V_{R T}} K_{V_{R T}} .
\end{gathered}
$$

In the above equations we have $\sigma_{0}=\sigma_{0}(u, \zeta, \bar{\zeta}), W_{0}=$ $W_{0}(u, r, \zeta, \bar{\zeta}), \Psi_{1}^{0}=\Psi_{1}^{0}(u, \zeta, \bar{\zeta})$ and

$$
\begin{aligned}
\Psi_{2}^{0}= & \Psi_{2}^{0}(u, \zeta, \bar{\zeta}) \\
& =-\left(M_{0}+\lambda\left(M_{1}(u, \zeta, \bar{\zeta})+i \mu(u, \zeta, \bar{\zeta})\right)\right) ;
\end{aligned}
$$

where $\mu$ is related to $\sigma_{0}$ by

$$
\mu=\frac{1}{2 i}\left(\check{\partial}_{V_{R T}}^{2} \bar{\sigma}_{0}-\bar{\partial}_{V_{R T} \sigma_{0}}^{2} .\right.
$$

In order to study the intrinsic fields at future null infinity (scri), it is convenient to consider the leading order behavior of $W_{0}$, namely

$$
W_{0}=\frac{\Psi_{0}^{0}}{4 ! r}+W_{1} ;
$$

where $\Psi_{0}^{0}=\Psi_{0}^{0}(u, \zeta . \bar{\zeta})$ and $W_{1}=O\left(1 / r^{2}\right)$. It is interesting to note that the component $\Psi_{0}=\frac{\partial^{4} W_{0}}{\partial r^{4}}$ of the Weyl tensor is given in this case 24 by

$$
\Psi_{0}=\frac{\Psi_{0}^{0}}{r^{5}}+\frac{\partial^{4} W_{1}}{\partial r^{4}},
$$

where the second term is of order $O\left(1 / r^{6}\right)$.

The remaining equations at scri are:

$$
\dot{\Psi}_{0}^{0}=3 \frac{\dot{V}_{R T}}{V_{R T}} \Psi_{0}^{0}+\mathrm{\partial}_{V_{R T}} \Psi_{1}^{0}-3 M_{0} \sigma_{0},
$$

$$
\dot{\Psi}_{1}^{0}=3 \frac{\dot{V}_{R T}}{V_{R T}} \Psi_{1}^{0}-\breve{\partial}_{V_{R T}}\left(M_{1}+i \mu\right)-\bar{\partial}_{V_{R T}}\left(K_{V_{R T}}\right) \sigma_{0}
$$

and

$$
\begin{aligned}
& -6 M_{0} \frac{\dot{V}_{\lambda}}{V_{R T}}=\bar{\varpi}_{V_{R T}} \partial_{V_{R T}} K_{V_{\lambda}}-6 \frac{\dot{V}_{R T}}{V_{R T}}\left(3 M \frac{V_{\lambda}}{V_{R T}}-M_{1}\right) \\
& -2 \dot{M}_{1}-\frac{\dot{V}_{R T}}{V_{R T}} \overline{\bar{\partial}}_{V_{R T}}^{2} \sigma_{0}+2 \frac{\dot{V}_{R T}}{V_{R T}^{2}} \bar{\partial}_{V_{R T}} V_{R T} \bar{\partial}_{V_{R T}} \sigma_{0} \\
& -\frac{2}{V_{R T}} \bar{\partial}_{V_{R T}} \dot{V}_{R T} \bar{\partial}_{V_{R T}} \sigma_{0}+\bar{\partial}_{V_{R T}}^{2} \dot{\sigma}^{0} \\
& -\frac{\dot{V}_{R T}}{V_{R T}} ð_{V_{R T}}^{2} \bar{\sigma}_{0}+2 \frac{\dot{V}_{R T}}{V_{R T}^{2}} \partial_{V_{R T}} V_{R T} \partial_{V_{R T}} \bar{\sigma}_{0} \\
& -\frac{2}{V_{R T}} \partial_{V_{R T}} \dot{V}_{R T} \partial_{V_{R T}} \bar{\sigma}_{0}+\widetilde{\partial}_{V_{R T}}^{2} \dot{\bar{\sigma}}^{0} .
\end{aligned}
$$

The objective is to use the solutions of the previous equations to model a black hole that just formed after the non head-on collision of a previous binary system. In this model the idea is to only make use of the information of the individual masses and the total angular momentum. With all this in mind we have to chose the appropriate gauge and free functions.

In reference 24] it was discussed the gauge freedom of these spacetimes. In order not to introduce extra structure, we will chose the free functions and gauges that make

$$
M_{1}=0
$$


and

$$
\sigma_{0}=0 .
$$

Other choices will force extra structure on the model beyond masses and angular momentum; as mentioned above.

With this choice the previous equations become

$$
\begin{gathered}
\dot{\Psi}_{0}^{0}=3 \frac{\dot{V}_{R T}}{V_{R T}} \Psi_{0}^{0}+\mathrm{\partial}_{V_{R T}} \Psi_{1}^{0}, \\
\dot{\Psi}_{1}^{0}=3 \frac{\dot{V}_{R T}}{V_{R T}} \Psi_{1}^{0}
\end{gathered}
$$

and

$$
-6 M_{0} \dot{V}_{\lambda}=V_{R T} \overline{\widetilde{\partial}}_{V_{R T}} \partial_{V_{R T}} K_{V_{\lambda}}-18 M_{0} \dot{V}_{R T}\left(\frac{V_{\lambda}}{V_{R T}}\right) .
$$

It is interesting to note that since the RT equation can also be expressed as

$$
-6 M_{0} \frac{\dot{V}_{R T}}{V_{R T}}=\bar{\partial}_{V_{R T}} \partial_{V_{R T}} K_{V_{R T}},
$$

equation (32) can be written in the following way

$$
\begin{aligned}
&-6 M_{0} \dot{V}_{\lambda}=V_{R T} \bar{\partial}_{V_{R T}} \partial_{V_{R T}} K_{V_{\lambda}} \\
&+3 V_{\lambda} \bar{\partial}_{V_{R T}} \partial_{V_{R T}} K_{V_{R T}} .
\end{aligned}
$$

Given initial conditions for the functions $V_{R T}, \Psi_{0}^{0}, \Psi_{1}^{0}$ and $V_{\lambda}$ equations (17), (30), (31) and (32) provide for the respective evolutions.

The radiation content of the spacetime is easily described by the time derivative of the Bondi shear. Let $\sigma_{B}^{0}$ denote the Bondi shear and $u_{B}$ the Bondi time; then for any section of scri $u=$ constant one can express the radiation content from the relation 29.

$$
\frac{\partial \sigma_{B}^{0}}{\partial u_{B}}=\frac{\partial^{2} V}{V} ;
$$

therefore to first order one has

$$
\frac{\partial \sigma_{B}^{0}}{\partial u_{B}}=\frac{\partial^{2} V_{R T}}{V_{R T}}+\lambda\left(\frac{\partial^{2} V_{\lambda}}{V_{R T}}-\frac{V_{\lambda} \partial^{2} V_{R T}}{V_{R T}^{2}}\right) .
$$

The radiation flux at the retarded time $u$ is given by

$$
F_{B}(u)=\frac{1}{4 \pi} \int_{S_{u}} \frac{\partial \sigma_{B}^{0}}{\partial u_{B}} \frac{\partial \bar{\sigma}_{B}^{0}}{\partial u_{B}} d S^{2} .
$$

Let us recall that in this case the timelike component of the Bondi momentum at the section $u=$ constant can be calculated from

$$
P_{B}^{0}=\frac{1}{4 \pi} \int \frac{M_{0}}{V^{3}} d S^{2} .
$$

At the retarded time $u$, we say that $V$ represents a quadrupole excitation along the $y$ axis if

$$
V=1+A Y_{2,0}\left(\zeta^{\prime}, \bar{\zeta}^{\prime}\right)
$$

where $A$ is the amplitude of the excitation, $Y_{2,0}$ is an spherical harmonic, and $\left(\zeta^{\prime}, \bar{\zeta}^{\prime}\right)$ are the coordinates of the sphere where the pole is along the $y$ axis. Then in terms of the usual spherical harmonics with coordinates $(\zeta, \bar{\zeta})$, one has

$$
V=1-\frac{A}{2}\left(\sqrt{\frac{3}{2}} Y_{2,-2}(\zeta, \bar{\zeta})+Y_{2,0}(\zeta, \bar{\zeta})+\sqrt{\frac{3}{2}} Y_{2,2}(\zeta, \bar{\zeta})\right) .
$$

\section{$3 \quad$ Fixing the parameters and the Newtonian matching}

The physical system can be characterized by two stages. During the first stage two black holes are moving towards each other with some orbital angular momentum. At some moment they collide and form a single black hole; which settles down in the asymptotic future to a stationary Kerr geometry.

During the first stage of evolution we describe the gravitational radiation with the quadrupole formula, where the dynamics is worked out from the Newtonian framework. The whole motion is contained in a plane; therefore two variables are sufficient to describe the orbits. The two integrals of motion, namely energy $E$ and angular momentum $j$, allow to solve the Newtonian system.

The initial data is assumed to be given in the $(x, y)$ plane; with zero total momentum and such that the orbital angular momentum is along the positive $\hat{z}$ direction

The initial velocities can be thought to have components along the $x$ axis only. Let us call $R_{0}$ the initial impact parameter, at some initial relative distance $r_{0}$. In this way, using the relative velocity $v_{0}$, one has

$$
E=\frac{\mu}{2} v_{0}^{2}-\frac{G m_{1} m_{2}}{r_{0}},
$$

$$
j=\mu R_{0} v_{0} ;
$$

where $\mu=\frac{m_{1} m_{2}}{m_{1}+m_{2}}$ is the reduced mass.

For this kind of motion the quadrupole formula predicts that the power of gravitational radiation is given by

$$
F_{Q}(r)=\frac{8}{15}\left(G m_{1} m_{2}\right)^{2}\left[\frac{2}{\mu r^{4}}\left(E+\frac{G m_{1} m_{2}}{r}\right)+\frac{11 j^{2}}{\mu^{2} r^{6}}\right] ;
$$

which generalizes the analog equation appearing in ref. 22 .

In order to continue the dynamical description after the collision of the two black holes, we need to have a merging condition in the Newtonian framework. In references 21] and 22] we have succeeded in estimating the total energy radiated in the head on black hole collision using the following criteria. When a black hole, which 
mass at infinity is $m_{i 1}$, is brought to a distance $r_{12}$ of another black hole of asymptotic mass $m_{i 2}$, its physical mass, for the stationary situation, is changed 30 to

$$
m 1=m_{i 1}+\frac{m_{i 1} m_{i 2}}{2 r_{12}} ;
$$

and similarly for the other mass.

Applying the arguments of ref. 22] one concludes that the merging condition should be taken when the separation distance has the value

$$
r_{c}=2(m 1+m 2) .
$$

It is convenient to introduce the relative mass parameter $\alpha$ and the reference mass $m$, so that $m_{i 1}=m$ and $m_{i 2}=\alpha m$. Then the merging condition 22 gives

$$
r_{c}=\left(m_{i 1}+m_{i 2}\right)\left[1+\sqrt{1+\frac{2 \alpha}{(1+\alpha)^{2}}}\right] .
$$

Let us now consider the initial data used in ref. [2] for the grazing collisions of black holes; namely: $x_{1}=5 \mathrm{~m}$, $x_{2}=-5 m, y_{1}=m, y_{2}=-m, v_{x 1}=-0.5$ and $v_{x 2}=0.5$; with $m=1$ and $\alpha=1$. This data corresponds to a hyperbolic Newtonian trajectory; with initial separation distance $r=10.198$, and critical merging radius $r_{c}=$ 4.449 .

Our strategy is to follow closely the model used in 21. 22; but we also want to compare our work with [2]. However, the initial data of [2] involves two black holes with half the speed of light each; therefore in adapting this initial data to our model we must take into account relativistic effects. In references [21] and [22] there was no need for these concerns because the initial data was not relativistic.

Since the initial velocities are relativistic, the relative initial velocity $v_{0}$ is calculated from the expression

$$
v_{0}=\frac{v_{x 2}-v_{x 1}}{1-v_{x 1} v_{x 2}}=0.8 ;
$$

where we are using geometric units in which the velocity of light and the gravitational constant have the unit value.

The energy radiated during the falling phase, calculated from (43) is $E_{N}=0.00346\left(m_{1}+m_{2}\right)$.

In order to match the Newtonian stage with the black hole RT perturbed model we need also to set the total initial mass and initial angular momentum for the RT stage.

In previous work we have matched the Newtonian mass $M_{N e w}=m_{i 1}+m_{i 2}$ to the initial mass $M$; this already takes into account the field relativistic first order correction in terms of its physical masses $m 1$ and $m 2$, since, recalling equation (21) of ref. 22, the initial mass would be $m 1+m 2-\frac{m_{i 1} m_{i 2}}{r_{12}}$. The system discussed in ref. 22 had zero initial velocity, and therefore there was no need to take into account any other effect. Instead in our case we should take into account speed relativistic corrections.

Then, since the initial data is relativistic, the initial mass and angular momentum are calculated from

$$
M=\frac{m_{i 1}}{\sqrt{1-v_{1}^{2}}}+\frac{m_{i 2}}{\sqrt{1-v_{2}^{2}}}=2.309
$$

and

$$
J=-y_{1} \frac{m_{i 1} v_{x 1}}{\sqrt{1-v_{x 1}^{2}}}-y_{2} \frac{m_{i 2} v_{x 2}}{\sqrt{1-v_{x 2}^{2}}}=1.155 .
$$

It should be emphasized that the relation between the initial RT stage mass and angular momentum $M$ and $J$ with the Newtonian mass and angular momentum $M_{N e w}$ and $j$ is just the Lorentzian factor $\gamma=\frac{1}{\sqrt{1-v_{x 1}^{2}}}=1.1547$. In the last section we will comment on the incidence of this factor on our results.

Let us observe that the relation between the relativistic angular momentum and total mass is $\frac{J}{M^{2}}=0.217$.

Since the angular momentum is small, it can be treated as a perturbation. At the moment of the collapse, we can consider a quadrupole excitation along the $y$ axis with amplitude $A$, as described above. Let us take $A=A_{m}+A_{j}$; where $A_{j}$ is the contribution coming from the appearance of the angular momentum $j$. The matching condition is given by the equation (See equations (35), (37) and (43).)

$$
F_{B}=F_{Q}\left(r_{c}\right)
$$

from which one obtains in this case

$$
A_{m}=0.057
$$

and

$$
A_{j}=0.028 .
$$

Therefore, to be explicit, the initial data for $V_{R T}$ and $V_{\lambda}$ are

$$
\begin{aligned}
& V_{R T}=1-\frac{A_{m}}{2} \\
& \times\left(\sqrt{\frac{3}{2}} Y_{2,-2}(\zeta, \bar{\zeta})+Y_{2,0}(\zeta, \bar{\zeta})+\sqrt{\frac{3}{2}} Y_{2,2}(\zeta, \bar{\zeta})\right)
\end{aligned}
$$

and

$$
\begin{aligned}
& \lambda V_{\lambda}=-\frac{A_{j}}{2} \\
& \times\left(\sqrt{\frac{3}{2}} Y_{2,-2}(\zeta, \bar{\zeta})+Y_{2,0}(\zeta, \bar{\zeta})+\sqrt{\frac{3}{2}} Y_{2,2}(\zeta, \bar{\zeta})\right) .
\end{aligned}
$$

The constant $M_{0}$ is determined from (38), and the condition that initially the mass is given by (48); which sets $M_{0}=2.302$. 
The orbital angular momentum is taken into account in the initial data for $\Psi_{1}^{0}$. It is convenient to express this initial data in terms of the auxiliary field $g$, given by

$$
\Psi_{1}^{0}=i \frac{\partial_{V_{R T}} g}{V_{R T}^{2}}=i \frac{\partial g}{V_{R T}} .
$$

Let us note that then

$$
\check{\partial}_{V_{R T}} \Psi_{1}^{0}=\check{\partial}^{2} g
$$

so that in the stationary case one has $\widetilde{\partial}^{2} g=0$ and $\dot{g}=0$.

We take $g=\bar{g}$ and

$$
g=g_{0} Y_{1,0}(\zeta, \bar{\zeta})
$$

where $g_{0}$ is related to the angular momentum in the $z$ direction by

$$
g_{0}=-\sqrt{12 \pi} J=-4.094 .
$$

In reference 31] it was also considered a similar case of a binary system with orbital angular momentum. Their initial data was: $x_{1}=0, x_{2}=0, y_{1}=m, y_{2}=-m$, $v_{x 1}=-0.8$ and $v_{x 2}=0.894$; with $m=1.5$ and $\alpha=\frac{2}{3}$. From the Newtonian point of view this data corresponds to an elliptic motion; but with maximum and minimum radius that are smaller than the corresponding critical merging radius. For this reason, we can not compare this case with our model.

\section{Numerical Implementation}

Accurate numerical evolution of a fourth order parabolic equation, such as (17), by means of an explicit finite difference scheme is a challenge because the $C F L 32$ condition requires that the time step $\Delta u$ scale as the fourth power of the spatial grid size. Nevertheless, we constructed a set of algorithms to solve these equations using second order accurate finite difference approximations (following [33). The numerical treatment of the eth operator has been thoroughly described in [33. This work presented a clean way to deal with derivative operators on the sphere by covering it with two coordinate patches and dealing with spin weighted quantities. Thus, it is ideally suited for our present purposes. The numerical grid on each patch is defined by $\xi_{i j}=q_{i}+i p_{j}$ where $q_{i}, p_{i}=-1-2 \Delta_{A}+(i-1) \Delta_{A}\left(\right.$ with $\left.\Delta_{A}=2 /\left(N_{A}-5\right)\right)$. The angular derivatives are discretized by centered second order finite difference approximations and information between patches is obtained through fourth order accurate interpolations. (For a detailed description of this approach see 33 ).

The integration in time is based upon a three time level Adams-Bashford 32 scheme with predictor $(\tilde{\mathcal{F}})$ given by

$$
\tilde{\mathcal{F}}(u+\Delta u)=\mathcal{F}(u)+\frac{\Delta u}{2} \partial_{u}[3 \mathcal{F}(u)-\mathcal{F}(u-\Delta u)]
$$

and corrector

$$
\begin{aligned}
\mathcal{F}(u+\Delta u)=\mathcal{F}(u)+\frac{\Delta u}{2} \partial_{u}[\mathcal{F}(u)+ & \tilde{\mathcal{F}}(u+\Delta u)] \\
& +O\left(\Delta u^{3}\right)
\end{aligned}
$$

Where $\mathcal{F}$ stands for $V_{R T}$ or $V_{\lambda}$ and the $\partial_{u}$ terms are to right hand sides of equations (27). Additionally, we implemented the iterative Cranck-Nicholson algorithm 34, 35 and observed that the results obtained with both implementations agree. Since the evolution equation for $\Psi_{0}^{0}$ is linear, its numerical integration is straightforwardly done by centered second order differences at the level $(u+\Delta u / 2)$.

The second order convergence of numerical solutions was confirmed in the perturbative regime using solutions of the linearized equation and second order selfconvergence of the solutions was confirmed in the nonlinear regime.

\section{Results}

\section{Variations of total energy}

The total Bondi energy-momentum vector at any RT retarded time $u$ can be calculated from the expression

$$
P^{\mathrm{a}}=-\frac{1}{4 \pi} \int_{S} l^{\mathrm{a}}(\zeta, \bar{\zeta})\left(\Psi_{B 2}^{0}+\sigma_{B} \dot{\bar{\sigma}}_{B}\right) d S^{2},
$$

where $S$ is the section determined by $u=$ constant,

$$
\left(l^{\mathrm{a}}\right)=\left(1, \frac{\zeta+\bar{\zeta}}{1+\zeta \bar{\zeta}}, \frac{\zeta-\bar{\zeta}}{i(1+\zeta \bar{\zeta})}, \frac{\zeta \bar{\zeta}-1}{1+\zeta \bar{\zeta}}\right) ;
$$

and the subscript $B$ is used to emphasize that the quantities are evaluated with respect to a Bondi frame. The mass $M$ at this section $S$ is then given by

$$
M=\sqrt{P^{a} P_{a}},
$$

where the indices are raised and lowered by the Lorentzian flat metric $\eta_{a b}$ at scri 36.

Let us note that the relations between the Bondi quantities and the intrinsic ones are

$$
\Psi_{B 2}^{0}=\frac{\Psi_{2}^{0}}{V^{3}}
$$

and

$$
\sigma_{B}=\frac{\sigma}{V}
$$

therefore in our gauge one has $\sigma_{B}=0$, at each RT section.

The gravitational energy radiation flux is calculated from the Bondi time derivative of the supermomentum $\Psi$ 37]; namely

$$
\frac{\partial \Psi}{\partial u_{B}}=\frac{\partial \sigma_{B}}{\partial u_{B}} \frac{\partial \bar{\sigma}_{B}}{\partial u_{B}} .
$$


If one instead considers the time change with respect to the RT time, it is convenient to have in mind that for any function $f$ one has

$$
\frac{\partial f}{\partial u_{B}}=\frac{1}{V} \frac{\partial f}{\partial u}
$$

The so called news function $\frac{\partial \sigma_{B}}{\partial u_{B}}$ can be expressed in terms of the perturbed RT fields by

$$
\frac{\partial \sigma_{B}}{\partial u_{B}}=\frac{\partial^{2} V}{V}+\frac{1}{V^{2}}\left(\dot{\sigma}-\frac{\dot{V}}{V} \sigma\right) .
$$

To calculate the total energy radiated, one could then numerically evaluate the gravitational energy radiation flux of equation (65) at different times and sum along all the elapsed time. However, it is more accurate to numerically evaluate the initial mass and subtract the final mass. This is due to the fact that the RT spacetime is known to converge asymptotically to the Schwarzschild one; more specifically, one knows that $\lim _{u \rightarrow \infty} V_{R T}=1$; and similarly one can see that $\lim _{u \rightarrow \infty} V_{\lambda}=0$.

Using this procedure, and a resolution of $n=32$ points for half a meridian of the sphere (approximately $N=1600$ points for the whole sphere), the energy radiated $E_{R T}$ during the RT stage is found to be $E_{R T}=$ $0.0034 M_{0}$.

Then, since in units of $M_{0}$, the energy radiated in the first stage is $E_{N}=0.0030 M_{0}$, the total energy radiated in the whole process is $E=0.0064 M_{0}$.

\section{Gravitational radiation field}

The numerical calculation of the evolution of the gravitational radiation field $\Psi_{4}$, is shown in figure $\mathbb{1}$.

It can be seen that although the total energy radiated is rather small, the amplitude of the gravitational radiation field can be large. In other words, this model describes a noticeable burst.

This is interesting since $\Psi_{4}$ is precisely what gravitational wave detectors will measure.

\section{Variations of angular momentum}

When dealing with the notion of angular momentum one is faced with the fact that there are several inequivalent definitions of angular momentum; which are not tightly related with the notion of intrinsic angular momentum, with the exception of [38]. An appropriate definition of intrinsic angular momentum involves the selection of unique sections 38 of future null infinity where the quantity is to be calculated. Since in our case we are taking the angular momentum as a perturbation parameter of the RT geometries, it is not essential to consider these refinements in our model. And also, since the RT spacetimes provide with a geometric unique family of sections of future null infinity, namely the sections $u=$ constant, it is natural to use them to calculate the angular momentum.

Then, instead of describing the variation of the intrinsic angular momentum we describe the variation of the
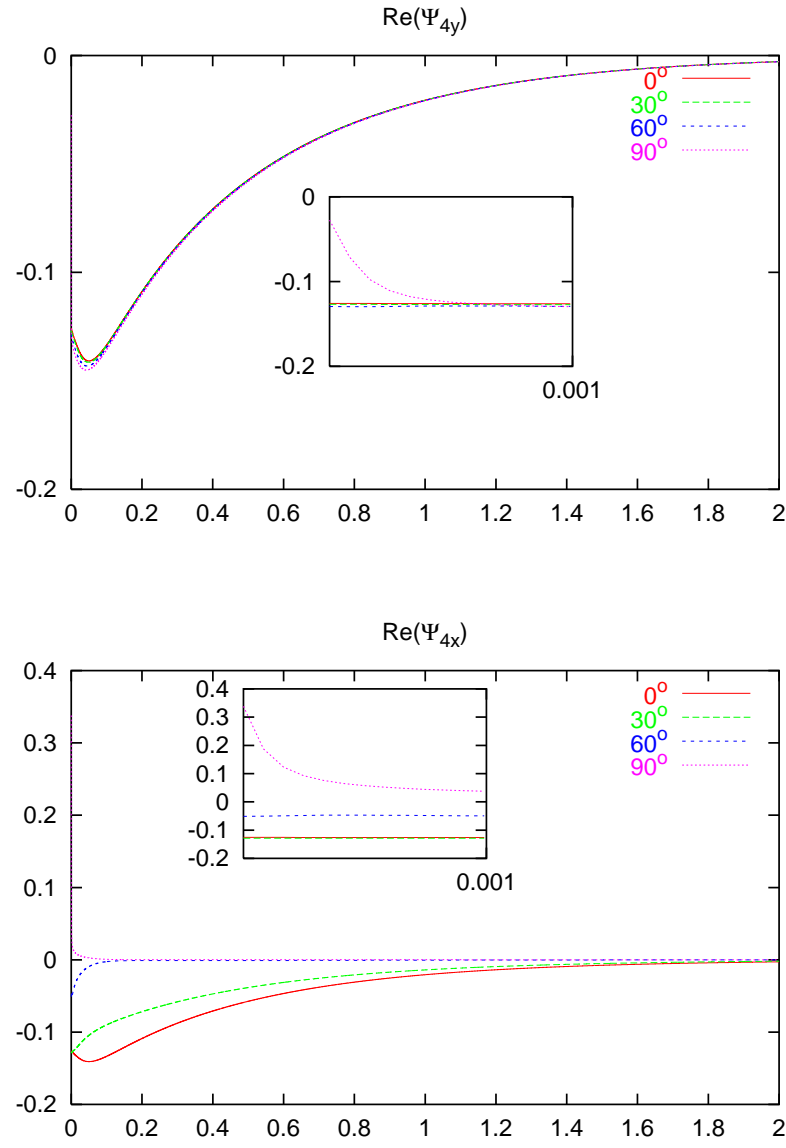

Figure 1: Evolution of $\Psi_{4}$ as a function of time. The subscript $x$ and $y$ refer to the $x$ and $y$ axis of the stereographic coordinate $\zeta=x+i y$ of the northern hemisphere. Each of the four curves refer to detectors at $0^{\circ}, 30^{\circ}, 60^{\circ}$ and $90^{\circ}$; measured from the north pole of the sphere. The insets show the detail of the rapid time variations at the beginning.

RT-angular momentum vector given by

$$
J^{k}=\Re \mathrm{e}\left[\int_{S_{R T}} \frac{i}{4 \pi} \overline{\bar{\partial}} \ell^{k} \Psi_{B 1}^{0} d S_{B}^{2}\right]
$$

where $S_{R T}$ are the sections determined by $u=$ constant, $k=1,2,3$, so that $\ell^{k}$ are the spacelike components of $\ell^{\mathrm{a}}$; and where the Bondi component $\Psi_{B 1}^{0}$, of the Weyl tensor, is related to the RT Weyl component $\Psi_{1}^{0}$ by

$$
\Psi_{B 1}^{0}=\frac{\Psi_{1}^{0}}{V^{3}}
$$

Figure 2 shows a very small and smooth variation of the angular momentum; which is more related to the time variation of the RT geometry than to the radiation of angular momentum; as can be seen from the nature of equation (31). 


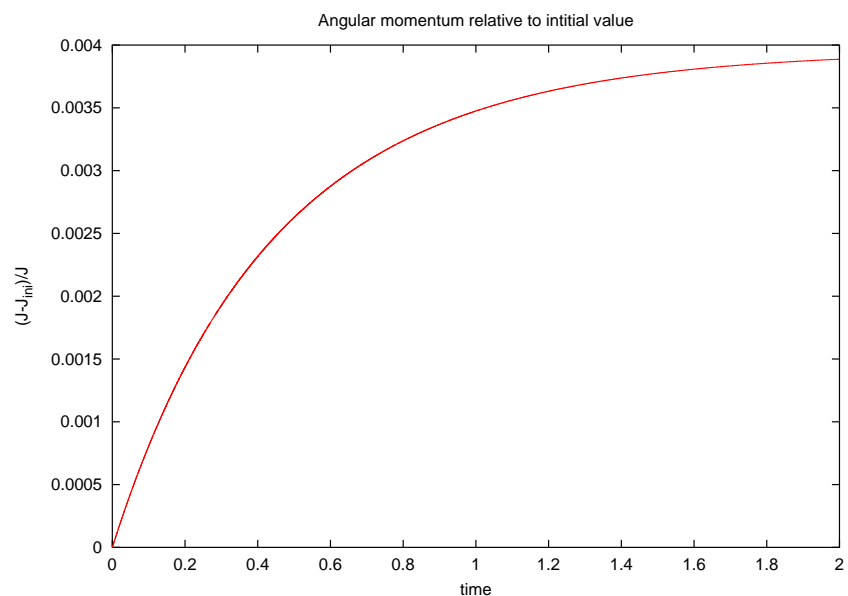

Figure 2: Evolution of the relative variation of angular momentum $J$ as a function of time.

\section{Final comments}

The estimate of the total gravitational energy radiated in the non head-on collision of two black holes is less than one percent according to our model. This is on the low end value of existing numerical results (which are still being refined) and comparable with those obtained with the close limit approximation[39. It is however worth mentioning that our calculations predict an amplitude of the gravitational waves that could be important from the observational point of view. Additionally, it predicts a rather narrow time duration of the 'burst'. Since the frequency at which this happens; its amplitude and the time duration can be used as preliminary information for constructing data analysis 'filters' 40, 41, knowledge of these is of importance while templates from full numerical simulations are not available. We will carry out a detailed study of these in the future.

At first sight there is too large a difference between the numerical results in [2] and the estimates obtained here. However, one should keep in mind several points which (by themselves) can account for this difference. Comments are in place for both the results in [2] and those presented here:

First, the results in [2] were obtained under a rather coarse resolution and the final numbers need to be refined (there are intense efforts worldwide in this direction). Additionally, the obtained value of radiated energy come from comparisons of initial ADM mass and apparent horizon masses. Masses obtained from apparent horizon calculations, in dynamical regimes, are only an approximation of the mass the black hole and hence results obtained through this method can have a significant systematic error. Furthermore it is important to remember that the initial data of [2] is given at a spacelike hypersurface of the spacetime; while, by the nature of the RT spacetime, our initial data is given on a characteristic surface. This implies an essential difference since in the spacelike hypersurface initial data one has incoming and outgoing radiation contributing to the total energy radiated; while in the $\mathrm{RT}$ characteristic problem that we solve, we do not consider any incoming initial radiation in our calculations. So, for the relativistic regime, one expects the time slice initial data to radiate more energy than the characteristic case.

As far as the present model is concerned: in previous works we have obtained very good agreements between our estimates and the mature numerical calculations of exact geometries; as one can check in the family of systems depicted in figures 3 and 4 of reference [22]. The fact that the model represented so well such a variety of initial data and different mass ratios, motivated us to apply the same techniques to the case in which angular momentum was involved. However, since there are just two (still being refined) numerical calculations available to compare with, it is difficult to obtain much information at this stage.

As we have mentioned before our aim is to follow as close as possible the model used in [21] and [22]; however the fact that the initial conditions considered are relativistic posse several questions. For example, in matching the masses of the two stages we have used equation (48); so one might ask how would the results change if the relativistic $\gamma$ factor was not used? This would certainly decrease the initial $M$ and $M_{0}$ by about $13 \%$; but $E_{N}$ and $E_{R T}$ would not change, so that the ration between the total energy radiated versus the changed $M_{0}$ would increase by the factor $\gamma$, that is about $15 \%$.

Similarly, had we not used the relativistic correction on the angular momentum $J$, its initial value would have decreased by about 13\%; but the relative variation shown in figure 2 would not have changed.

In any case, we think that by applying our model to this relativistic initial data, we are pushing the model to the boundary of its validity. Comparisons with future numerical simulations will shed light on this and indicate how far this model can be pushed.

The radiation of angular momentum seems to be negligible with these initial conditions. In order to consider higher values of the initial angular momentum, we would need to deal with other background geometries, as for example twisting algebraically special spacetimes. Regarding the smooth monotonic variation of it one can infer that, for this small initial angular momentum data, its behavior is driven by the exponential asymptotic behavior of the RT background geometry. There are not complicated initial variations in $\Psi_{1}^{0}$, that for example do appear in $\Psi_{4}^{0}$, as seen in figure 11.

When describing a concrete physical situation with these spacetimes, one is supposed to choose the gauge and fix the free functions in order to make the best representation of the system. It is somehow striking that the choice of the frame in first order has physical significance, and it is not pure gauge as one is accustomed in the studies of linearized gravity around Minkowski 
spacetime.

\section{Acknowledgments}

We acknowledge support from CONICET, SeCyT-UNC, NSF Grants No. PHY9900791 and PHY0090091, and Eberly Research Funds of Penn State. Some computations were performed in the VN cluster (vn.physics.ubc.ca) supported by the Canadian Foundation for Innovation.

We are grateful to an anonymous Referee for suggesting several improvements and pointing out some typos.

\section{References}

[1] Luis Lehner. Class. Quant. Grav., 18:R25-R86, 2001.

[2] Steve Brandt et al. Phys. Rev. Lett., 85:5496-5499, 2000 .

[3] Lawrence E. Kidder, Mark A. Scheel, and Saul A. Teukolsky. Phys. Rev., D64:064017, 2001.

[4] Miguel Alcubierre, Bernd Brugmann, Denis Pollney, Edward Seidel, and Ryoji Takahashi. Phys. Rev., D64:061501, 2001.

[5] H. J. Yo, T. W. Baumgarte and S. L. Shapiro, Phys. Rev. D 64, 124011 (2001).

[6] Bernard Kelly et al. Phys. Rev., D64:084013, 2001.

[7] R. Gomez, S. Husa and J. Winicour. Phys. Rev., D64:024010, 2001.

[8] L. Blanchet. Living Reviews, (2002).

[9] C. M. Will, Prog. Theor. Phys. Suppl. 136, 158 (1999)

[10] L. Blanchet, G. Faye, B Iyer and B. Joguet, Phys. Rev., D59:061501, 2002.

[11] A. Buonanno and T. Damour. Phys. Rev., D59:084006, 1999

[12] K. Thorne, Gravitational Radiation - A New Window Onto the Universe, gr-qc/9704042. 1997.

[13] L.Grishchuk, V.M.Lipunov, K.A.Postnov, M.E.Prokhorov and B.S.Sathyaprakash. Phys.Usp. 44 (2001) 1-51.

[14] Richard H. Price and Jorge Pullin. Phys. Rev. Lett. 72:3297-3300, 1994.

[15] J. Pullin, Prog. Theor. Phys. Suppl. 136, 107 (1999) arXiv:gr-qc/9909021.

[16] I. Robinson and R. Trautman. Proc. R. Soc. A, 265:463-473, 1962.
[17] K. P. Tod. Class. Quantum Grav., 6(8):1159-1163, 1989.

[18] P. T. Chruściel, D. B. Singleton, Commun. Math. Phys. 147, 137 (1992).

[19] L. Derry, R. Isaacson and J. Winicour, Phys. Rev. 185, 1647 (1969).

[20] O. M. Moreschi. Approximating vacuum asymptotic field equations around stationary spacetimes. GR16, Workshop A4, Durban, South Africa, 2001; oral presentation.

[21] O. M. Moreschi and S. Dain. Phys. Rev. D, 53(4):R1745-R1749, 1996. Rapid Communication.

[22] O. M. Moreschi. Phys. Rev. D, 59:084018, 1999.

[23] Peter Anninos, David Hobill, Edward Seidel, Larry Smarr, and Wai-Mo Suen. Phys. Rev., D52:20442058, 1995.

[24] O. M. Moreschi and A. Perez. Class. Quantum Grav., 18:3701-3720, 2001.

[25] E. Newman and R. Penrose, J. Math. Phys. 7, 863 (1966).

[26] J. N. Goldberg et al., J. Math. Phys. 8, 2155 (1967).

[27] R. Penrose and W. Rindler, Spinors and SpaceTime, Vol. 1, (Cambridge University Press, Cambridge, 1984).

[28] R. Geroch and A. Held and R. Penrose, J. Math. Phys., 14, 874-881, (1973).

[29] S. Dain, O. M. Moreschi, and R. J. Gleiser. Class. Quantum Grav., 13(5):1155-1160, 1996.

[30] D. Brill. Phys.Rev., 131:471, 1963.

[31] B. Bruegmann, Annalen Phys. 9, 227, (2000).

[32] B. Gustafsson, H.-O. Kreiss and J. Oliger, Time Dependent Problems and Difference Methods, (Wiley Interscience, New York, 1995).

[33] R. Gomez, L. Lehner, P. Papadopoulos and J. Winicour, Class. Quant. Grav. 14, 977 (1997).

[34] S. Teukolsky, Phys. Rev. D 61, 087501 (2000).

[35] M. Alcubierre, et. al. Phys. Rev. D 62, 044034 (2000).

[36] O. M. Moreschi. Class. Quantum Grav., 3:503-525, 1986.

[37] O. M. Moreschi. Class. Quantum Grav., 5:423-435, 1988. 
[38] O. M. Moreschi. Center of mass frame, angular momentum and asymptotic structure equations. GR16, Workshop A3, Durban, South Africa, 2001; oral presentation.

[39] R. Gleiser, G. Khanna, R. Price and J. Pullin, New Jour.Phys. 2, 3 (2000).

[40] W. G. Anderson, P. Brady, J. Creighton and E. Flanagan. Phys. Rev., D63:042003, 2001.

[41] J. Creighton, Phys. Rev., D60:021101, 1999. 